\title{
Anticonvulsant effect of exogenous $\beta$-hydroxybutyrate on kainic acid-induced epilepsy
}

\author{
JIANPING SI ${ }^{1,2}$, SHAOHUI WANG ${ }^{3}$, NING LIU ${ }^{4}$, XIAOFEI YANG ${ }^{5}$, \\ YING WANG ${ }^{6}, \mathrm{LING} \mathrm{LI}^{4}$, JIWEN WANG ${ }^{1}$ and $\mathrm{XIN} \mathrm{LV}^{4}$ \\ ${ }^{1}$ Department of Neurology, Children's Medical Center, Qilu Hospital of Shandong University, \\ Brain Science Research Institute, Shandong University, Jinan, Shandong 250012; \\ ${ }^{2}$ Department of Pediatrics, The People's Hospital of Guangrao, Dongying, Shandong 257300, P.R. China; \\ ${ }^{3}$ Department of Molecular Pharmacology and Physiology, University of South Florida College of Medicine, Tampa, \\ FL 33606, USA; ${ }^{4}$ Institute of Pediatric Research, Qilu Children's Hospital of Shandong University, Jinan, Shandong 250022; \\ ${ }^{5}$ Department of Pediatrics, Yidu Central Hospital, Weifang, Shandong 262500; \\ ${ }^{6}$ Department of Pediatrics, Jinan Central Hospital Affiliated to Shandong University, Jinan, Shandong 250013, P.R. China
}

Received January 31, 2016; Accepted February 10, 2017

DOI: $10.3892 /$ etm.2017.4552

\begin{abstract}
The present study aimed to investigate the anticonvulsant effects of $\beta$-hydroxybutyrate (BHB) in a kainic acid (KA)-induced rat seizure model. The concentrations of BHB and glucose were detected in the blood prior to exogenous BHB administration. Three different doses of BHB (2, 4 and $8 \mathrm{mmol} / \mathrm{kg}$ ) were then injected into male Wistar rats intraperitoneally, and the concentrations of BHB and glucose in the blood were detected. Rats received $0.9 \%$ normal saline intraperitoneally at a dose of $4 \mathrm{ml} / \mathrm{kg}$ as a control. Subsequently, a KA-induced rat seizure model was established and the anticonvulsant effects of BHB were investigated. The onset time of seizure and the degree of seizure behavior were recorded. Nissl and Timm staining were used to evaluate neuronal loss and mossy fiber sprouting, respectively. The present study demonstrated that exogenous BHB administration could significantly increase BHB concentration in the blood and this concentration was maintained for 90 min without affecting blood glucose levels. Furthermore, it was determined that a dose of $4 \mathrm{mmol} / \mathrm{kg}$ BHB is optimal for exogenous administration. The onset time of seizure was significantly prolonged in BHB-pretreated rats $(63.31 \pm 4.050 \mathrm{~min})$ compared with
\end{abstract}

Correspondence to: Dr Jiwen Wang, Department of Neurology, Children's Medical Center, Qilu Hospital of Shandong University, Brain Science Research Institute, Shandong University, 107 Wenhuaxi Road, Jinan, Shandong 250012, P.R. China

E-mail: wjiwwang@sina.com

Dr Xin Lv, Institute of Pediatric Research, Qilu Children's Hospital of Shandong University, 430 Jingshi Road, Jinan, Shandong 250022, P.R. China

E-mail: lvxinetyy@163.com

Key words: ketogenic diet, $\beta$-hydroxybutyrate, kainic acid, epilepsy the control group $(37.08 \pm 1.958 \mathrm{~min} ; \mathrm{P}=0.039)$. In addition, neuronal loss and mossy fiber sprouting were both alleviated in the BHB-pretreated model group. Exogenous BHB administration at a dose of $4 \mathrm{mmol} / \mathrm{kg}$ may be an alternative to a ketogenic diet to exert a protective effect in the epileptic model induced by KA. The results of the present study may allow novel therapeutic strategies to be developed to treat epilepsy.

\section{Introduction}

Epilepsy is a chronic neurological disease, characterized by recurrent epileptic seizures, and causes impairments in neurobiology, cognition, psychology and social behavior $(1,2)$. Multiple antiepileptic drugs are available; however, $230 \%$ of patients with epilepsy experience undesirable adverse reactions and develop resistance to these drugs $(3,4)$.

The ketogenic diet (KD) is a high-fat, low-carbohydrate and moderate-protein diet. It has anticonvulsant and anti-epileptogenic effects, including epileptogenesis inhibition and neuronal loss prevention, in amygdala-kindling seizures (5) and is considered to be an effective treatment for medically refractory epilepsy $(6,7)$. However, the mechanisms underlying its clinical efficacy have not been elucidated. Increasing evidence supports that $\beta$-hydroxybutyrate (BHB) induced by KD may increase the concentration of $\gamma$-aminobutyric acid (GABA) in the epileptic brain by inhibiting astrocytic GABA degradation (6). Additionally, it has been reported that levels of BHB in the blood are positively correlated with seizure resistance (8). Abdelmalik et al (9) observed that pretreatment with $\mathrm{BHB}$ reduced the frequency of seizures induced by acute hypoglycemia. Furthermore, exogenous BHB administration may prolong the onset time of seizure in an epilepsy model induced by pilocarpine and flurothyl $(10,11)$. However, the dose of BHB administered varies in different studies (9-11), suggesting that the dose of exogenous BHB may be a key factor for epilepsy therapy. Additionally, the association between blood $\mathrm{BHB}$ levels and the dose of $\mathrm{BHB}$ administered remains unclear. A 
path analysis demonstrated that the seizure threshold is significantly elevated with increasing ketogenic ratios, but not with increasing BHB levels in rats (12). Therefore, determining the optimal dose of BHB administration that is close to the $\mathrm{BHB}$ levels in rats following KD may optimize the anticonvulsant and anti-epileptogenic effects of BHB in epilepsy.

The aim of the present study was to investigate the association between BHB levels in the blood and the BHB exogenous dosage, and to investigate the anticonvulsant effects of exogenous BHB on the rat seizure model induced by kainic acid (KA). In addition, Nissl and Timm staining were used to evaluate the histological changes in KA-induced seizure models. The results of the present study may facilitate the development of novel therapeutic strategies to treat epilepsy.

\section{Materials and methods}

Animals. The present study was approved the Ethics Committee of Shandong University School of Medicine (Shandong, China). All experimental procedures were conducted according to the National Institute of Health Guidelines (13).

A total of 102 male Wistar rats on postnatal 21 days were obtained from Shandong University Animal Center (Jinan, China), weighing $60 \pm 10 \mathrm{~g}$. Rats had free access to food and tap water and housed at a standard temperature $\left(22 \pm 1^{\circ} \mathrm{C}\right)$ and humidity $(50 \pm 5 \%)$ under a 12-h light/dark cycle (lights on from 07:00 a.m. to 07:00 p.m.). Rats continued to be kept in the standard housing conditions until the time of the experiment. To detect the concentration of $\mathrm{BHB}, 32$ rats were divided into the following groups: BHB treatment $(2,4$ and $8 \mathrm{mmol} / \mathrm{kg}$; $\mathrm{n}=9$ in each) and normal saline (NS) control $(n=5)$. A total of 20 rats were used to detect the glucose concentration, treated with either $4 \mathrm{mmol} / \mathrm{kg}$ of BHB or $4 \mathrm{ml} / \mathrm{kg} \mathrm{NS}$ ( $\mathrm{n}=10$ in each group). To explore the anticonvulsant effect of $\mathrm{BHB}$ on KA-induced seizure model, a total of 50 rats were divided into $\mathrm{BHB}+\mathrm{KA}$ and NS+KA groups ( $\mathrm{n}=25$ in each group).

BHB and glucose concentration detection. DL-BHB (cat. no. H6501, Sigma-Aldrich; Merck KGaA, Darmstadt, Germany) was dissolved in sterile $0.9 \%$ NS at a concentration of $1 \mathrm{~mol} / \mathrm{l}$. Wistar rats on postnatal day 21were intraperitoneally administered with different concentrations of BHB (2, 4 and $8 \mathrm{mmol} / \mathrm{kg}$, respectively; $\mathrm{n}=9$ in each BHB group) immediately. Rats $(n=5)$ used as a control were administered with NS intraperitoneally at a dose of $4 \mathrm{ml} / \mathrm{kg}$. The serum obtained from these rats was used for detecting the concentration of BHB. In addition, another 20 Wistar rats on postnatal day 21 were intraperitoneally administered with $4 \mathrm{mmol} / \mathrm{kg}$ of $\mathrm{BHB}$ and $4 \mathrm{ml} / \mathrm{kg}$ NS ( $\mathrm{n}=10$ in each group) and their serum was used for detecting the concentration of glucose concentration.

At $0,15,45,75$ and 90 min after BHB administration, blood was collected from angular veins of the rats in above groups under ether anesthesia (Sigma-Aldrich; Merck KGaA). After the last time of blood collection, the rats were sacrificed immediately by decapitation under ether anesthesia. A total of $2 \mathrm{~h}$ after collection at room temperature, the blood was centrifuged at $3,000 \mathrm{x}$ g for $15 \mathrm{~min}$ at $4^{\circ} \mathrm{C}$ to obtain serum.

The concentration of BHB in the serum was then detected using a BHB assay kit (cat. no. MAK041, Sigma-Aldrich; Merck $\mathrm{KGaA}$ ) according to the manufacturer's protocol.
Glucose concentration in the serum was tested using a glucose meter (Roche Diagnostics, Basel, Switzerland).

Establishment of KA-induced rat seizure model. To induce seizure, 50 rats received intraperitoneal injection of $\mathrm{KA}[10 \mathrm{mg} / \mathrm{kg}$ in $0.9 \% \mathrm{NaCl}$, (pH 7.0), cat. no. K0250, Sigma-Aldrich; Merck KGaA] on postnatal day 21. The seizure behavior of animals was then analyzed $1 \mathrm{~h}$ after KA injection for $2 \mathrm{~h}$ according to the scale devised by Racine (13): Stage I, facial clonus; Stage II, head nodding and wet dog shaking; Stage III, forelimb clonus; Stage IV, forelimb with rearing; Stage V, rearing, jumping and falling. If 3 consecutive behaviors at each stage appeared, rats were scored. Then $10 \%$ chloral hydrate (400 mg/kg; Sigma-Aldrich; Merck KGaA) was administered intraperitoneally to stop seizure behavior if the status epilepticus continued over $90 \mathrm{~min}$. All rats presented with seizure behavior above stage IV and were considered to be a successful epileptic model.

Anticonvulsant effect of BHB on KA-induced seizure model. Another 50 Wistar rats were randomly selected and divided into $\mathrm{BHB}+\mathrm{KA}$ and $\mathrm{NS}+\mathrm{KA}$ groups ( $\mathrm{n}=25$ each group). Rats in the $\mathrm{BHB}+\mathrm{KA}$ group were administered with $4 \mathrm{mmol} / \mathrm{kg}$ BHB intraperitoneally $30 \mathrm{~min}$ prior to $\mathrm{KA}$ injection. Rats in the NS+KA group were administered with NS intraperitoneally prior to KA injection, and this group was used as a control. The onset time of stage IV or $\mathrm{V}$ and the degree of seizure behavior were recorded for $2 \mathrm{~h}$ following KA administration. Seizure behaviors of the rats in each group were observed and evaluated by an observer blind to the grouping.

Histological examination. Rats were anesthetized with $10 \%$ chloral hydrate $(400 \mathrm{mg} / \mathrm{kg})$ and their skulls were then immediately cut open for obtaining brains at 3 or 14 days following $\mathrm{KA}$ administration ( $\mathrm{n}=5$ at each time point). Brains were fixed in $4 \%$ paraformaldehyde for $24 \mathrm{~h}$ at $4^{\circ} \mathrm{C}$ and then embedded in paraffin. Coronal paraffin sections $4-\mu \mathrm{m}$ thick were prepared for staining. The remaining rats were housed under the conditions outlined above for use in future studies.

Nissl staining was performed to observe neuronal loss and damage in the CA1 and CA3 regions of the hippocampus in NS- and BHB-treated rats 3 days following KA administration. Coronal sections on day 3 were dewaxed, rinsed in crystal violet stain for $1 \mathrm{~h}$ at $56^{\circ} \mathrm{C}$ and heated for $10 \mathrm{~min}$. Sections were then immediately rinsed in distilled water, immersed in Nissl staining solution (Arcturus Bioscience, Inc., Mountain View, CA) for $3 \mathrm{~min}$, dehydrated in absolute ethyl alcohol, cleared in xylene and mounted with neutral gum solution. Typical neuronal loss and damage in the CA1 and CA 3 region following treatment were observed under a microscope (Nikon $80 \mathrm{i}$, Nikon Corporation, Tokyo, Japan) at a magnification of $\mathrm{x} 50$ and $\mathrm{x} 400$.

Timm staining was performed to observe mossy fiber sprouting (MFS) in NS- and BHB-treated rats 14 days following KA administration. Coronal sections on day 14 were stained in Timm staining solution (120 $\mathrm{ml} 50 \%$ arabic gum, $20 \mathrm{ml}$ citric acid buffer, $30 \mathrm{ml} \mathrm{57 \%}$ hydroquinone and $30 \mathrm{ml}$ 0.73\% silver lactate; all Sigma-Aldrich; Merck KGaA) in the dark at $26^{\circ} \mathrm{C}$ for $90 \mathrm{~min}$. Sections were then washed with distilled water, dehydrated and mounted with neutral gum 
solution. MFS was evaluated by rating the granule distribution in the dentate gyrus and CA3 region in Timm staining. Timm staining scale ranged between 0 and 5 following these criteria: 0 , No granules; 1 , sporadic granules in a patchy distribution; 2, more granules in patchy distributions; 3 , granules in a continuous distribution with occasional patches; 4 , dense granules in a near-continuous laminar band and 5, dense granules in a continuous laminar band. Coronal sections were observed under a microscope (Nikon 80i, Nikon Corporation) at a magnification of $\mathrm{x} 100$ and $\times 200$.

Statistical analysis. Statistical analyses were performed using SPSS software 20.0 (IBM SPSS, Armonk, NY, USA). All data were expressed as the mean \pm standard error of the mean, obtained from at least three independent experiments. The differences of the BHB concentration, glucose concentration and average Timm staining score were evaluated using one-way analysis of variance and further comparison between groups was performed by post hoc Tukey test. Differences in the onset time and degree of seizure behavior were evaluated using the Student's t-test and $\mathrm{P}<0.05$ was considered to indicate a significant difference.

\section{Results}

Exogenous $B H B$ administration significantly increases the concentration of $\mathrm{BHB}$ in the blood. Prior to $\mathrm{BHB}$ administration, the concentration of $\mathrm{BHB}$ in the blood was $\sim 0.57 \pm 0.01 \mathrm{mmol} / 1$. The concentration of BHB in the blood increased to $1.35-2.37 \mathrm{mmol} / 115 \mathrm{~min}$ after BHB administration, and this level was maintained for the next 75 min (Fig. 1). Compared with the $\mathrm{NC}$ group, BHB concentrations were significantly higher in $\mathrm{BHB}$ groups $(\mathrm{P}<0.05)$ at all time points; however, there was no significant difference in the blood BHB concentration between the rats administrated with 2, 4, or $8 \mathrm{mmol} / \mathrm{kg} \mathrm{BHB}$. Notably, it was observed that the BHB concentration in the blood was relatively stable at $1-2 \mathrm{mmol} / 1$ after the rats were administrated $4 \mathrm{mmol} / \mathrm{kg}$ BHB (Fig. 1), which was most similar to the BHB levels after rats had been on KD (14). There were significant differences among different times in $4 \mathrm{mmol} / \mathrm{kg}$ BHB group compared with the control group $(\mathrm{P}=0.020$ at $15 \mathrm{~min}$ and $\mathrm{P}<0.001$ at 45,75 and $90 \mathrm{~min}$ ). Thus, $4 \mathrm{mmol} / \mathrm{kg} \mathrm{BHB}$ was used to treat rats in the subsequent analyses.

Exogenous BHB administration has no significant effect on blood glucose. The concentration of glucose in the blood initially increased over 15 min following BHB administration and then decreased in the subsequent next 75 min (Fig. 2). However, there were no significant differences in glucose concentrations compared with the control group $(\mathrm{P}=0.702$, $0.398,0.350,0.891,0.838$ at $0,15,45,75,90 \mathrm{~min}$, respectively; Fig. 2). Additionally, there were also no significant differences in the glucose concentration among different times in each group $(\mathrm{P}=0.246$ in the $\mathrm{BHB}$ and $\mathrm{P}=0.333$ in the NS groups; Fig. 2).

Onset time of seizure is prolonged in a KA-induced seizure model following $B H B$ pretreatment. The onset time of seizure in the $\mathrm{BHB}+\mathrm{KA}$ group was $63.31 \pm 4.050 \mathrm{~min}$, which was

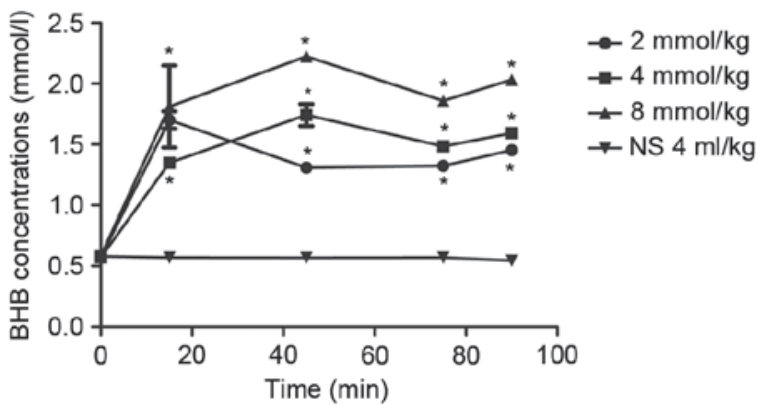

Figure 1. Exogenous BHB administration increases its concentration in the blood. BHB concentration in the blood increased rapidly within $15 \mathrm{~min}$ and was maintained in the next $75 \mathrm{~min}$ at all doses $(2,4$ and $8 \mathrm{mmol} / \mathrm{kg})$ of exogenous BHB. There was no significant difference in the blood BHB concentration for differnet doses of BHB. Notably, the BHB concentration in the blood was relatively stable at $1-2 \mathrm{mmol} / \mathrm{l}$ after the rats were administrated with $4 \mathrm{mmol} / \mathrm{kg} \mathrm{BHB}$, which was similar to the BHB levels when rats are on KD. ${ }^{*} \mathrm{P}<0.05$ vs. control group. BHB, $\beta$-hydroxybutyrate; KD, ketogenic diet; NS, normal saline.

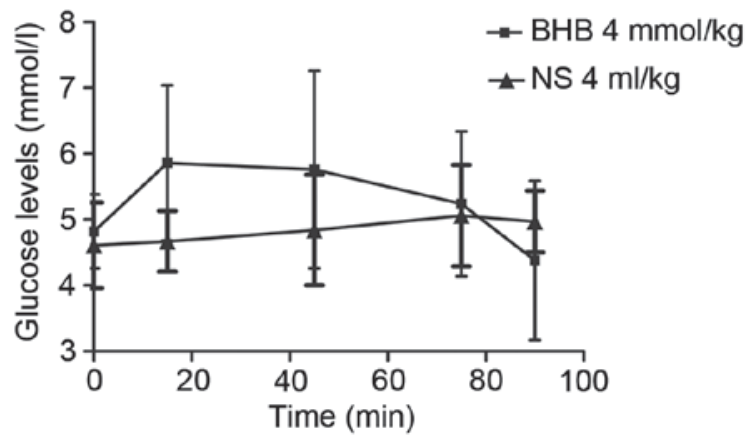

Figure 2. Exogenous BHB administration had no significant effect on blood glucose levels. Following exogenous BHB administration, the glucose level gradually increased over $15 \mathrm{~min}$ and then decreased in the next $75 \mathrm{~min}$. However, there was no significant difference between the two groups $(\mathrm{P}=0.702,0.398,0.350,0.891$ and 0.838 at $0,15,45,75$ and $90 \mathrm{~min}$, respectively). There was also no significant difference in the glucose concentration among the different times in the same group $(\mathrm{P}=0.246$ in the $\mathrm{BHB}$ and $\mathrm{P}=0.333$ in the NS group). BHB, $\beta$-hydroxybutyrate; NS, normal saline.

significantly longer $(\mathrm{P}=0.039)$ than that of the $\mathrm{NS}+\mathrm{KA}$ group $(37.08 \pm 1.958 \mathrm{~min}$; Fig. 3). Furthermore, the average degree of seizure behavior in the BHB+KA group was $4.54 \pm 0.100$, which was slightly lower than that in the NS+KA group (4.67 \pm 0.098$)$, however, this difference was not significant ( $\mathrm{P}=0.069$; Fig. 4).

Neuronal loss in the hippocampus and MFS is alleviated in a BHB-pretreated KA-induced seizure model. The results of Nissl staining demonstrated that there was no neuronal loss in the hippocampus in NS- and BHB-treated rats on day 3. However, typical neuronal loss and damage in the CA1 and $\mathrm{CA} 3$ region were found in the KA-induced seizure model that did not receive $\mathrm{BHB}$ pretreatment. Furthermore, neuronal loss was attenuated in the $\mathrm{BHB}+\mathrm{KA}$ group compared with the NS+KA group (Fig. 5).

MFS in each group on day 14 was observed using Timm staining. The results demonstrated that the average Timm score in KA-induced seizure rats was significantly higher than that in the NS- and BHB-treated rats $(\mathrm{P}=0.005)$ Furthermore, there was no significant difference in the average Timm score 


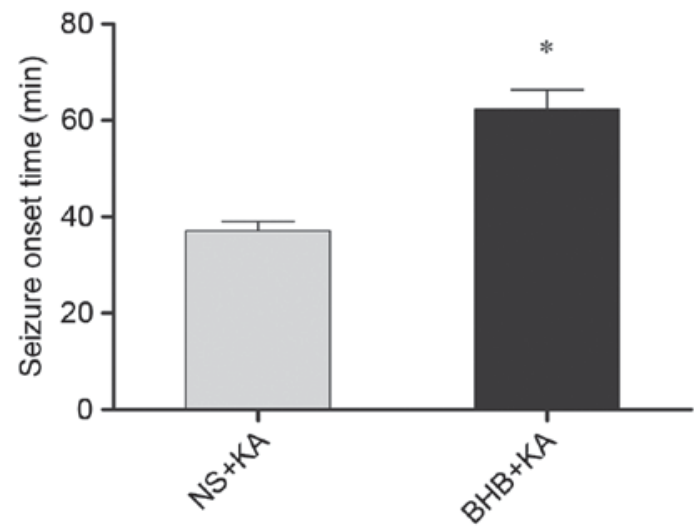

Figure 3. Exogenous BHB administration prolonged the onset time of seizure. The onset time of seizure in the BHB-pretreated group was $63.31 \pm 4.050 \mathrm{~min}$, which was significantly longer than that of the control group $(37.08 \pm 1.958 \mathrm{~min}) .{ }^{*} \mathrm{P}=0.039$ vs. Control group. BHB, $\beta$-hydroxybutyrate; KA, kainic acid; NS, normal saline.

between the NS $(0.13 \pm 0.06)$ and BHB groups $(0.23 \pm 0.06$; $\mathrm{P}=0.183$; Fig. 6). Compared with the NS group (3.67 \pm 0.15$)$, the average Timm score of the BHB+KA group $(1.5 \pm 0.50)$ was significantly decreased $(\mathrm{P}=0.021$; Fig. 6), indicating that MFS was alleviated in the KA-induced seizure model group receiving BHB pretreatment.

\section{Discussion}

Epilepsy is a chronic illness and $\sim 30 \%$ of patients with epilepsy are refractory to current pharmacotherapies (15). Thus, it is important to identify more effective therapies to treat patients with epilepsy. Studies have determined that exogenous BHB is neuroprotective and acts as an anticonvulsant in vitro and in vivo $(16,17)$. Therefore, the present study investigated the anticonvulsant effect of $\mathrm{BHB}$ and the results demonstrated that exogenous BHB could increase blood BHB concentration, but had no evident effect on blood glucose. Exogenous BHB administration could also increase the concentration of BHB in the blood to a similar level observed in rats treated with KD (14) and this concentration could be maintained for $90 \mathrm{~min}$. Furthermore, the onset time of seizure was significantly prolonged while neuronal loss and MFS were attenuated in BHB-pretreated rats with a KA-induced seizure.

$\mathrm{KD}$ is an established and effective therapy in the management of refractory epilepsy $(18,19)$. In vivo, KD can be metabolized into ketone bodies, including acetoacetic acid, $\mathrm{BHB}$ and acetone. Compared with acetoacetic acid and acetone, BHB has some advantages in that it is stable at physiological temperatures and can easily pass the blood-brain barrier (16). Additionally, the level of BHB in the blood can be altered by exogenous administration and BHB is considered to be preferable to treat patients with epilepsy, particularly for those with related metabolic abnormalities as it is a simple and safe method to induce elevated plasma levels of ketone bodies (16). It has also been reported that seizures occur more frequently if the blood glucose level is elevated during KD treatment (11). Meidenbauer and Roberts (14) demonstrated that acute glucose utilization would increase aberrant synchronous neuronal discharges, thus leading to seizure burst. The results of the

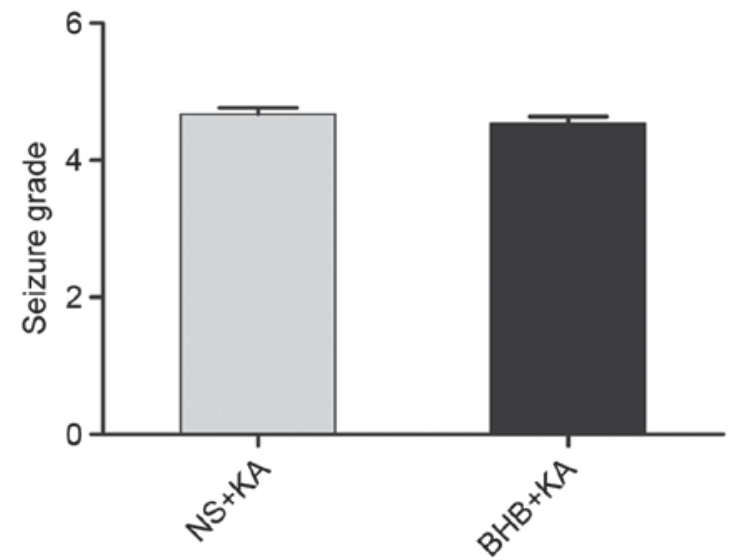

Figure 4. Degree of seizure behavior did not differ significantly in the BHB-pretreated model group following KA administration compared with the control. The average degree of seizure behavior in the BHB+KA group was $4.54 \pm 0.100$, which was slightly lower than that of the NS+KA group (4.67 \pm 0.098$)$. However, this difference was not significant $(\mathrm{P}=0.069)$. BHB, $\beta$-hydroxybutyrate; KA, kainic acid; NS, normal saline.

present study determined that exogenous administration of $4 \mathrm{mmol} / \mathrm{kg}$ BHB could increase blood BHB concentration, but had no effects on glucose levels, despite the fact that rats were fed a normal, unrestricted diet. This suggests that exogenous BHB administration does not cause an acute increase in glucose levels.

Furthermore, KD can increase the BHB level significantly and rats on KD had a significantly increased threshold for seizure induction (20). Furthermore, KD is not antiepileptic until BHB levels in the blood reach an efficacious level (1-2 mmol/l in rats) (14). In the present study, the concentration of BHB in the blood increased to an efficacious level just 15 min following exogenous BHB administration and this level was maintained for $90 \mathrm{~min}$, indicating that exogenous BHB administration may be a convenient and efficient way to elevate its concentration in the blood. If the BHB concentration in the cerebrospinal fluid was also tested at the corresponding time, the process of BHB utilization and metabolism following exogenous administration would be elucidated in more detail. Additionally, the onset time of seizures was prolonged in a KA-induced seizure model following BHB pretreatment in the present study, even though the degree of seizure behavior was not significantly decreased. This is consistent with previous findings demonstrating that $\mathrm{KD}$ increased the seizure threshold in rats but did not alleviate seizure severity (20). More experiments are still needed to verify the findings of the present study.

The present study also showed that neuronal loss and MFS were markedly diminished in the BHB-pretreated group compared with rats that did not undergo BHB pretreatment. MFS is thought to be epileptogenic and strongly associated with the occurrence of spontaneous recurrent seizure (21). Altogether, the similarity in the effects of BHB and KD on seizure susceptibility suggests that exogenous BHB may be an anticonvulsant alternative to KD. Patients on KD are only allowed a narrow range of foods, thus limiting patient food choices and permitted dishes may require long, complex preparation. Furthermore, adverse reactions such as nausea, vomit and diarrhea, experienced by some patients on KD 

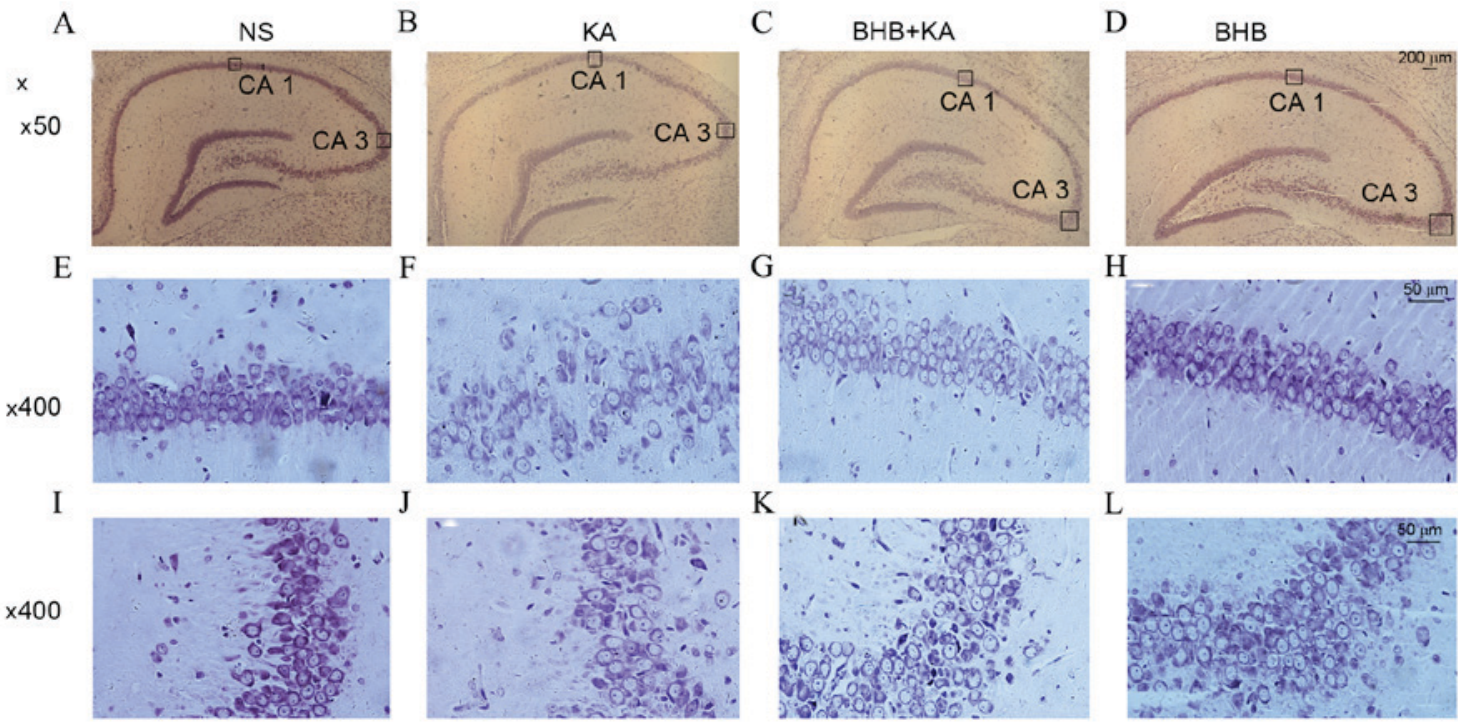

$\mathrm{L}$

$\times 400$
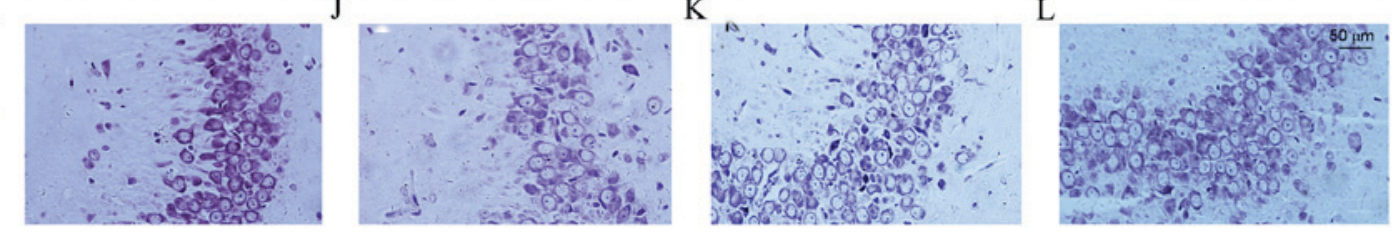

Figure 5. Nissl staining revealed the neuronal number in the hippocampus 3 days following KA administration. Typical neuronal loss and damage in the CA1 and CA3 region were observed in a KA-induced seizure model without BHB pretreatment. (A-D) Typical neuronal loss and damage in the CA1 and CA3 region, (E-H) CA1 and (I-L) CA3 regions. The neuronal loss was alleviated in the BHB+KA compared with that in the NS+KA group. BHB, $\beta$-hydroxybutyrate; KA, kainic acid; NS, normal saline.

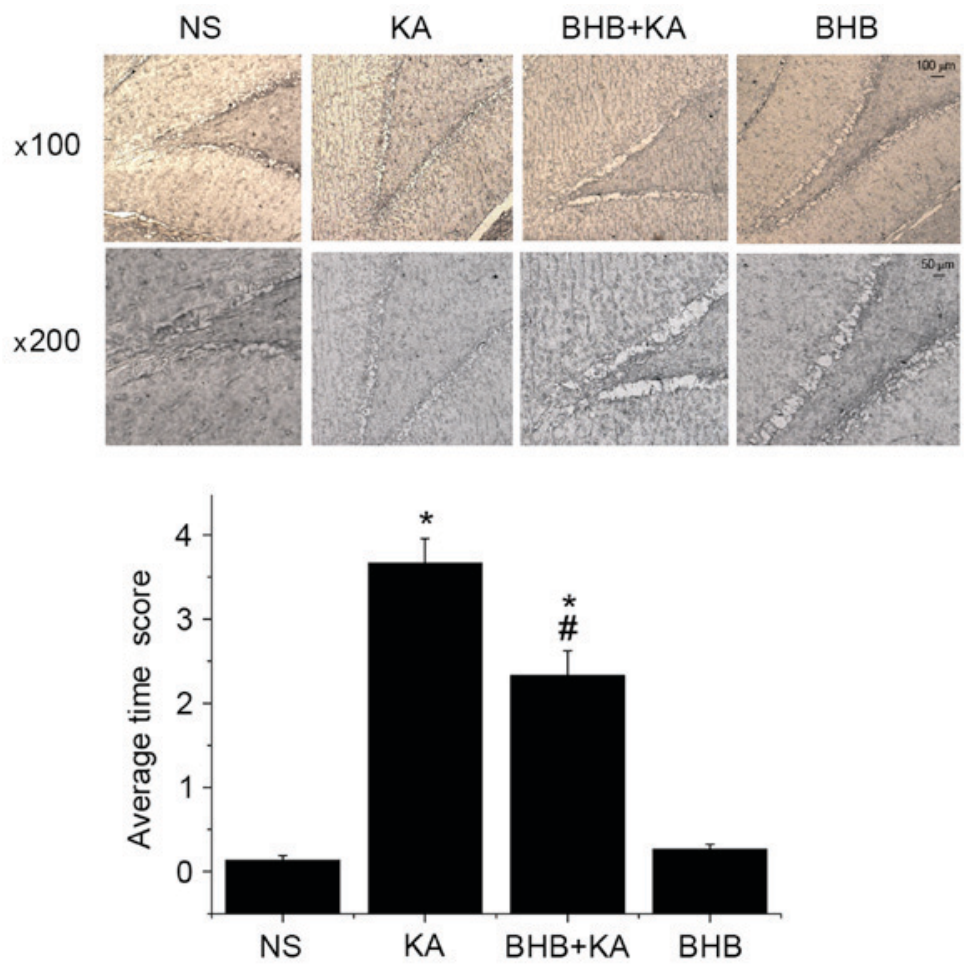

Figure 6. Timm staining presented CA3 and dentate gyrus inner molecular layer areas 14 days following KA administration. Timm scores in KA-induced seizure rats were significantly higher than that of NS- and BHB-treated rats. The average Timm score between the NS and BHB groups did not differ significantly. Compared with the NS group, the average Timm score of the BHB+KA group was significantly alleviated ( $\mathrm{P}=0.005$ vs. NS, ${ }^{*} \mathrm{P}=0.021 \mathrm{vs}$. KA; $\mathrm{n}=3$ in each group). BHB, $\beta$-hydroxybutyrate; KA, kainic acid; NS, normal saline.

have inhibited its application (22). Therefore, exogenous BHB preparations may be preferable to KD to treat epilepsy. Further studies are necessary to verify whether exogenous BHB administration may have a better therapeutic effect than KD in epilepsy treatment.

In conclusion, exogenous BHB administration at a dose of $4 \mathrm{mmol} / \mathrm{kg}$ could increase the BHB concentration in the blood without affecting blood glucose levels and increase the threshold of seizures, although it does not significantly the grades of seizure behavior. Additionally, exogenous BHB administration may attenuate the neuronal loss and MFS that occur in the hippocampus following convulsions. Exogenous BHB may be an alternative to KD to provide a protective effect in the epileptic model induced by KA. Therefore, the results of the present study may allow novel therapeutic techniques to be developed to treat epilepsy. 


\section{Acknowledgements}

The present study was supported by a project of the Shandong Province Science and Technology Program (grant no. 2014GSF118179) and the Special Foundation for Taishan Scholars (grant no. ts20110814).

\section{References}

1. Banerjee PN, Filippi D and Hauser WA: The descriptive epidemiology of epilepsy-a review. Epilepsy Res 85: 31-45, 2009.

2. Luan G, Zhao Y,Zhai F, Chen Y and Li T: Ketogenic diet reduces Smac/Diablo and cytochrome c release and attenuates neuronal death in a mouse model of limbic epilepsy. Brain Res Bull 89 79-85, 2012.

3. Kwan P and Brodie MJ: Early identification of refractory epilepsy. N Engl J Med 342: 314-319, 2000.

4. Wei C-X, Bian M and Gong GH: Current research on antiepileptic compounds. Molecules 20: 20741-20776, 2015.

5. Jiang Y, Yang Y, Wang S, Ding Y, Guo Y, Zhang MM, Wen SQ and Ding MP: Ketogenic diet protects against epileptogenesis as well as neuronal loss in amygdaloid-kindling seizures. Neurosci Lett 508: 22-26, 2012.

6. Suzuki Y,TakahashiH,Fukuda M,Hino H,Kobayashi K, Tanaka J and Ishii E: $\beta$-hydroxybutyrate alters GABA-transaminase activity in cultured astrocytes. Brain Res 1268: 17-23, 2009.

7. Gama IR, Trindade-Filho EM, Oliveira SL, Bueno NB Melo IT, Cabral-Junior CR, Barros EM, Galvão JA, Pereira WS, Ferreira RC, et al: Effects of ketogenic diets on the occurrence of pilocarpine-induced status epilepticus of rats. Metab Brain Dis 30: 93-98, 2015.

8. van Delft R, Lambrechts D, Verschuure P, Hulsman J and Majoie M: Blood beta-hydroxybutyrate correlates better with seizure reduction due to ketogenic diet than do ketones in the urine. Seizure 19: 36-39, 2010

9. Abdelmalik PA, Shannon P, Yiu A, Liang P, Adamchik Y, Weisspapir M, Samoilova M, Burnham WM and Carlen PL: Hypoglycemic seizures during transient hypoglycemia exacerbate hippocampal dysfunction. Neurobiol Dis 26: 646-660, 2007
10. Yum MS, Ko TS and Kim DW: $\beta$-Hydroxybutyrate increases the pilocarpine-induced seizure threshold in young mice. Brain Dev 34: 181-184, 2012.

11. Minlebaev M and Khazipov R: Antiepileptic effects of endogenous beta-hydroxybutyrate in suckling infant rats. Epilepsy Res 95: 100-109, 2011.

12. Bough KJ, Chen RS and Eagles DA: Path analysis shows that increasing ketogenic ratio, but not $\beta$-hydroxybutyrate, elevates seizure threshold in the rat. Dev Neurosci 21: 400-406, 1999.

13. National Research Council: Guide for the Care and Use of Laboratory Animals. 8 edition. Washington (DC), National Academies Press (US), 103: pp1072-1073, 2011.

14. Meidenbauer JJ and Roberts MF: Reduced glucose utilization underlies seizure protection with dietary therapy in epileptic EL mice. Epilepsy Behav 39: 48-54, 2014.

15. Löscher W: Current status and future directions in the pharmacotherapy of epilepsy. Trends Pharmacol Sci 23: 113-118, 2002.

16. Samoilova M, Weisspapir M, Abdelmalik P, Velumian AA and Carlen PL: Chronic in vitro ketosis is neuroprotective but not anti-convulsant. J Neurochem 113: 826-835, 2010.

17. Xie G, Tian W, Wei T and Liu F: The neuroprotective effects of $\beta$-hydroxybutyrate on $\mathrm{A} \beta$-injected rat hippocampus in vivo and in A $\beta$-treated PC-12 cells in vitro. Free Radic Res 49: 139-150, 2015.

18. Neal EG, Chaffe H, Schwartz RH, Lawson MS, Edwards N, Fitzsimmons G, Whitney A and Cross JH: The ketogenic diet for the treatment of childhood epilepsy: A randomised controlled trial. Lancet Neurol 7: 500-506, 2008

19. Henderson CB, Filloux FM, Alder SC, Lyon JL and Caplin DA Efficacy of the ketogenic diet as a treatment option for epilepsy: Meta-analysis. J Child Neurol 21: 193-198, 2006.

20. Bough KJ and Eagles DA: A ketogenic diet increases the resistance to pentylenetetrazole-induced seizures in the rat. Epilepsia 40: 138-143, 1999

21. Buckmaster PS, Zhang GF and Yamawaki R: Axon sprouting in a model of temporal lobe epilepsy creates a predominantly excitatory feedback circuit. J Neurosci 22: 6650-6658, 2002.

22. Giordano C, Marchiò M, Timofeeva E and Biagini G: Neuroactive peptides as putative mediators of antiepileptic ketogenic diets. Front Neurol 5: 63, 2014. 